Portland State University

PDXScholar

Electrical and Computer Engineering Faculty

Publications and Presentations

\title{
Modeling Acoustic Scattering from the Seabed Using Transport Theory
}

Jorge Quijano

Portland State University

Lisa M. Zurk

Portland State University, zurkl@pdx.edu

Follow this and additional works at: https://pdxscholar.library.pdx.edu/ece_fac

Part of the Acoustics, Dynamics, and Controls Commons, Oceanography and Atmospheric Sciences and Meteorology Commons, and the Remote Sensing Commons

Let us know how access to this document benefits you.

\section{Citation Details}

Quijano, J. E., \& Zurk, L. M. (2010, September). Modeling acoustic scattering from the seabed using Transport Theory. In OCEANS 2010 (pp. 1-6). IEEE.

This Conference Proceeding is brought to you for free and open access. It has been accepted for inclusion in Electrical and Computer Engineering Faculty Publications and Presentations by an authorized administrator of PDXScholar. Please contact us if we can make this document more accessible: pdxscholar@pdx.edu. 


\section{Modeling acoustic scattering from the seabed using Transport Theory}

\author{
Jorge E. Quijano \\ School of Electrical and \\ Computer Engineering \\ Portland State University \\ Portland, Oregon 97207-0751 \\ Email: jorgeq@pdx.edu
}

\author{
Lisa M. Zurk \\ School of Electrical and \\ Computer Engineering \\ Portland State University \\ Portland, Oregon 97207-0751 \\ Email: zurk1@cecs.pdx.edu
}

\begin{abstract}
Radiative Transfer (RT) theory has established itself as an important tool for electromagnetic remote sensing in parallel plane geometries with random distributions of scatterers, and most recently it has also been proposed as a model for the propagation of elastic waves in layered ocean sediments. In this work the capabilities of this model are illustrated, as the RT method is used to predict backscattering strength from laboratory models of random media. The RT model is characterized by its flexibility on accommodating scatterers in a broad variety of sizes, shapes, and acoustic contrast relative to the background media. Additionally, this formulation is easily expandable to include multiple layering and elastic effects. In this paper, a comparison between the RT model and the results from two laboratory experiments in the optics and the Mie regime are presented. The experiments were designed to measure volume scattering at high frequencies between $280 \mathrm{kHz}$ and 400 $\mathrm{kHz}$ in monostatic configuration. The first experiment used large aluminum spheres suspended with thin filaments, and it serves as a benchmark for testing the RT formulation due to its high signal-to-noise ratio, and the absence of reflective boundaries or background attenuation. Measurements of frequency dependent backscattering at normal incidence angle are shown for two fractional volumes. For the second experiment, the scattering media is a well characterized slab of a lossy resin background containing a uniform distribution of glass beads, and angle- and frequency-dependent measurements are presented. The levels of volume scattering from both experiments are found in agreement with predictions from the steady state RT model.
\end{abstract}

\section{INTRODUCTION}

Research on acoustic scattering from the seabed is of importance for shallow water active sonar and remote sensing applications, and great effort has been devoted to the development of mathematical models that explain the propagation and scattering of acoustic waves in media with such complexity [1]. Current models for rough surface and volume scattering are approximated solutions of the wave equation, valid under particular premises. For example, the assumptions of weak [2] or small scatterers [3] have been utilized to obtain a solution of the scattered field by the integral method. Other models use the assumption of high frequency to take advantage of poor sediment penetration, simplifying the mathematical complexity by ignoring the effects of deep layers. Nevertheless, compliance with the experimental conditions and parameters that justify such assumptions is not always possible and this might limit the range of applicability of a model. An alternative formulation widely used in electromagnetics and more recently in acoustics is Radiative Transfer (RT) theory [4], [5], which models the interaction of the intensity (as opposed to the acoustic field) with the scattering media by solving the so called transport equation. The goal of this paper is to present experimental evidence of the application of the RT model, by comparing simulations of volume scattering to measurements taken in a tank setup using well characterized scattering media.

Transport theory is a well known technique for remote sensing of the concentration of particles in the atmosphere [5]. More recently, research has been done to adapt the formulation to elastic waves, with potential applications in seismics [6], ultrasound [7] and seabed characterization [8]. The formal mathematical derivation of the RT model can be found in Twersky's theory of scattering from a random distribution of discrete inhomogeneities [4], and heuristic derivations based on the conservation of energy can also be found in the literature [5], [7]. The transport equation is a statement of the conservation of energy, cast in the form of and integrodifferential equation where the unknown quantity is the specific intensity, which can be related to the power flux. It has been suggested [5] that in general, given a system of discrete scatterers and an excitation source, solution methods for the scattered field are mathematically more tractable from the approach of transport theory than from classic wave theory. Another advantage of the RT model is that it provides more insight into the physical phenomena of scattering, by tracking the contribution of longitudinal and shear waves, background attenuation and the transformation from different polarizations at the boundaries of the scattering media as well as at the scatterers.

The algebraic details of the RT model for ocean bottom sediments have been presented in a previous publication [8] and therefore only a summary is included in this work. Additionally, a review of the relation between the specific intensity and received power is outlined [4] and used to compare RT simulations with volume scattering obtained in a laboratory setup.

The validation experiments for the RT model were per- 
formed in the optics $(k a>>1)$ and the Mie $(k a \approx 1)$ regimes, with lossless and lossy backgrounds, respectively. The experiment in the optics regime used aluminum spheres of radius $a=4.8 \mathrm{~mm}$, randomly arranged to fit within a volume representing a thin slab. Scattering measurements were taken for several concentrations of spheres, starting from a single sphere to test the calibration of the measurement system, to fractional volumes up to $2.7 \%$. The absence of reflective interfaces and background attenuation provided the optimal conditions to test the model with high signal-to-noise ratio, and it is shown that the RT model converges to known single scattering models [9].

The second experiment measured volume scattering from a more challenging scenario, using a lossy polyurethane slab with embedded glass beads. The background material has an acoustic impedance close to fresh water, with a lower compressional sound speed and a higher density. This results in the absence of a critical angle and favors energy penetration at all grazing angles. The slab does not support shear propagation, which is automatically accounted in the RT model by setting the shear sound speed to a small value of $1 \mathrm{~m} / \mathrm{s}$. The scattering material was manufactured to minimize the presence of unintended air bubbles, as corroborated by X-ray studies [10].

In both experiments, backscattered power was measured with a directional source and an omni directional receiver at ultrasonic frequencies, by exciting the media with long broadband pulses. Predictions obtained from the solution of the steady state RT equations are in agreement with the experimental data.

This paper is organized as follows: section II gives a summary of the relevant equations for transport theory, and the transformation of specific intensity into received power for comparison to experimental measurements. Section III is a description of the experimental methods and apparatus utilized in both experiments, followed by a comparison between experimental results and computations from the RT model in section IV. Section V corresponds to discussion of the results and conclusions.

\section{RADIATIVE TRANSFER MODEL}

The steady-state RT model for sediment scattering has been described in detail elsewhere [8], and therefore only a summary is presented in this section. To simplify the notation, and because the laboratory models used in the experiments do not support shear waves, the RT equations presented here only include energy of longitudinal polarization.

The RT model is well suited for layered environments with reflective boundaries and containing discrete scatterers, as illustrated in Fig. 1. Solutions of the volume scattering can be found for the bistatic system in Fig. 1(a), in which a directional source illuminates a finite patch denoted in blue. The size of this patch is determined by the radiation pattern of the transmitter and its distance from the media. An omni directional hydrophone will detect energy propagating within a solid angle that is entirely defined by the size of the illuminated patch and the radial distance to the slab. From Fig. 1(a), this solid angle is defined as $\Delta \Omega=d A / R^{2}$, where $d A$ is the area subtended by the solid angle at a distance $R$ from the receiver.

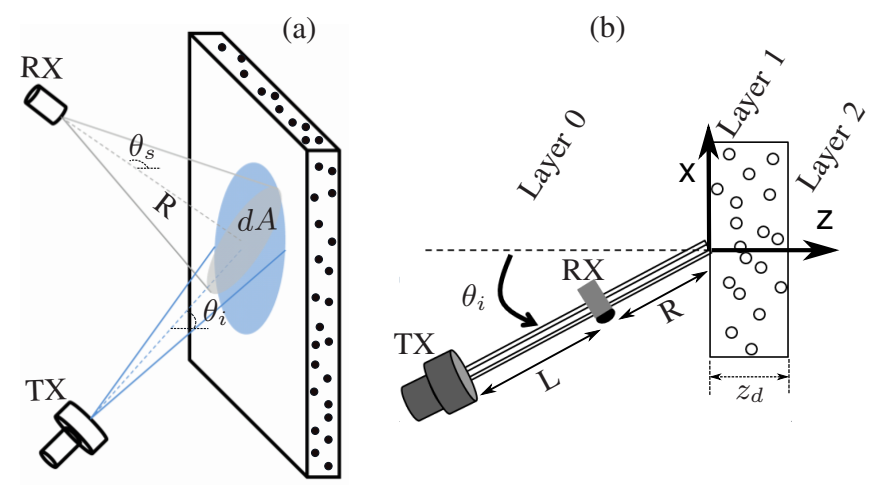

Fig. 1. Measurement of volume scattering from layered media: (a) General bistatic geometry in which a directional transmitter(TX) illuminates a patch (blue) defined by its radiation pattern and its distance from the media. The omni directional receiver detects the energy crossing the area $d A$ (gray); (b) Monostatic configuration used in the experiments presented in this paper. Values of $L$ and $R$ are given in section IV.

For the experiments presented in this paper, the measurement setup is the special case illustrated in Fig. 1(b), for which $\theta_{i}$ is the elevation angle that determines the direction of the excitation, while $\theta_{s}=\pi-\theta_{i}$ is the elevation corresponding to the scattered energy. The origin of the coordinate system is the center of the illuminated patch, with the $z$ axis pointing toward the media. Therefore, $\theta>0$ represents energy propagating from left to right. Without loss of generality the azimuth angle of the incident radiation is defined as $\phi_{i}=0^{\circ}$.

For a finite layer of thickness $z_{d}$ located between two halfspaces, the governing transport equation is given by

$$
\begin{array}{r}
\frac{\partial I(\mu, \phi, z)}{\partial(z / \mu)}+\frac{1}{c_{L 1}} \frac{\partial I(\mu, \phi, z)}{\partial t}=-[\eta(\kappa+\nu)+2 \alpha] I(\mu, \phi, z)+ \\
\frac{\eta}{4 \pi} \int_{-1}^{1} \int_{0}^{2 \pi} p\left(\mu, \phi ; \mu^{\prime}, \phi^{\prime}\right) I\left(\mu^{\prime}, \phi^{\prime}, z\right) d \mu^{\prime} d \phi^{\prime}
\end{array}
$$

where $\mu=\cos \theta$ and $\phi$ indicate the elevation and azimuth corresponding to the direction of propagation of the energy, $z$ is the depth, $\eta$ is the concentration of scatterers (number per cubic meter), $\kappa$ and $\nu$ are the scattering and absorption cross sections of a single scatterer, respectively; $\alpha$ is the background attenuation (in $\mathrm{Np} / \mathrm{m}$ ), $c_{L 1}$ is the longitudinal sound speed in layer 1 and $p\left(\mu, \phi ; \mu, \phi^{\prime}\right)$ is the phase matrix of a single scatterer, representing the redistribution of energy in a scattering event. The boundary conditions required to completely define the problem are given by:

$$
\begin{array}{r}
I(\mu, \phi, z=0)=\hat{R}_{10} I(-\mu, \phi, z=0) \\
+F \hat{T}_{10} \delta\left(\mu-\mu_{o}\right) \delta\left(\phi-\phi_{i}\right) \quad(0<\mu<1) ; \\
I\left(\mu, \phi, z=z_{d}\right)=\hat{R}_{12} I(-\mu, \phi, z=0) \quad(-1<\mu<0) ;
\end{array}
$$


where $\hat{R}_{10}$ and $\hat{R}_{12}$ are the reflection coefficients for the specific intensity at the interfaces between layers 1 and 0 and layers 1 and 2, respectively, and $\hat{T}_{10}$ is the transmission coefficient that couples the incident radiation of amplitude $F$ (in watt $/ \mathrm{m}^{2}$ ) into the slab. Note that the angle dependency of those coefficients has been omitted for brevity. The symbol $\delta$ is the Kronecker operator, and it is used in this context to indicate that the incident radiation is collimated in the direction given by $\left(\theta_{o}, \phi_{i}\right)$, where $\theta_{o}=\sin ^{-1}\left(\theta_{i} c_{L 1} / c_{L 0}\right)$ is the refracted angle into layer 1 given by Snell's law.

For the steady state form of the RT equation the time derivative is set to zero, and solution of (1) can be found by applying a Fourier series transformation to reduce the azimuth component, and a numerical method that divides the elevation component into $\mathrm{N}$ angles. This results in a NxN system of equations that can be solved by matrix inversions.

In oder to relate the solution of the RT model with experimental measurements, the power received at an omni directional hydrophone can be computed from the specific intensity $I(\mu, \phi, z)$ as:

$$
P_{r}=\int_{\Delta \Omega} \hat{T}_{10} I(\mu, \phi, z=0) d \Omega,
$$

where $\Delta \Omega$ was related to $d A$ in the discussion concerning Fig. 1(a). The integration in (3) can be solved numerically, but an approximation can provide some insight into the relation between the RT model and classic models. For example, in the far field $\Delta \Omega$ is small and it can be expected that $I(\mu, \phi, z=0)$ does not change significantly within the domain of integration, so it can be treated as a constant in the integration which reduces (3) to:

$$
P_{r}=\hat{T}_{10} I(\mu, \phi, z=0) \frac{d A}{R^{2}} .
$$

Then, the scattering cross section of the media is given by

$$
\Upsilon=R^{2} \frac{P_{r}}{P_{i}}=\hat{T}_{10} I(\mu, \phi, z=0) d A .
$$

Equation (5) has straightforward interpretation for special cases, such as small fractional volume or very thin slabs. For example, the single scattering solution of (1) (i.e. ignoring the integral term), with $\hat{R}_{10}=\hat{R}_{12}=\hat{T}_{10}=0$ is [4]:

$$
I(\mu, \phi, z=0)=\frac{\left|f\left(\mu, \phi, \mu_{o}, \phi_{i}\right)\right|^{2}}{\kappa} \frac{1-e^{\left[-\eta \kappa z_{d}\left(\frac{1}{\mu_{o}}-\frac{1}{\mu}\right)\right]}}{\mu_{o}-\mu} \mu_{o} F,
$$

where $\left|f\left(\mu, \phi, \mu_{o}, \theta_{i}\right)\right|$ is the scattering function of a single scatterer in the direction $(\mu, \phi)$ due to energy incident from the direction $\left(\mu_{o}, \phi_{i}\right)$. For thin layers or small fractional volumes, when $\left[-\eta \kappa z_{d}\left(\frac{1}{\mu_{o}}-\frac{1}{\mu}\right)\right]<<1,(5)$ reduces to

$$
\Upsilon=\left|f\left(\mu, \phi, \mu_{o}, \phi_{o}\right)\right|^{2} \eta z_{d} d A,
$$

which is the incoherent superposition of the returns from all the illuminated scatterers. The product $\eta z_{d}$ has been interpreted as a surface distribution in other models [9]. As observed here, the RT formulation automatically converges to this solution, but it is still applicable when the experimental conditions are more restrictive. The next section describes the setup for the two experiments mentioned in the introduction.

\section{EXPERIMENTAL SETUP}

Experiments in the optics and the Mie regimes were performed using the configuration shown in Fig. 1(b). In this section, the common features between both experiments are described, and further details specific to each experiment are provided in the next section.

All the measurements were performed in a rectangular tank of dimensions $5 \times 7 \times 3$ feet tall shown in Fig. 2, filled with fresh water with a measured sound speed $c_{L 0}=1468 \mathrm{~m} / \mathrm{s}$.

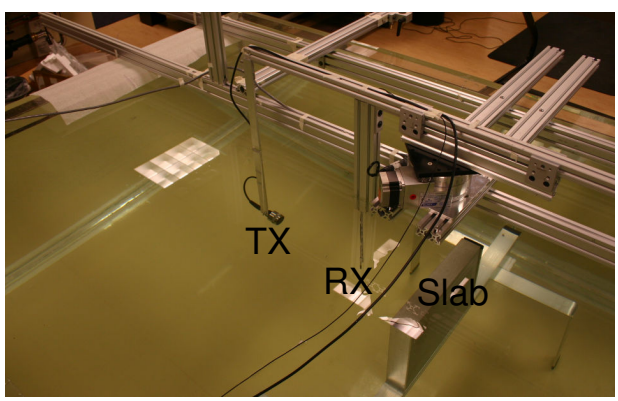

Fig. 2. Picture of the water tank, transmitter (TX), hydrophone (RX), and rotational mechanism used in the experiments. The resin slab used in experiment 2 can be seen held in position by a supporting aluminum structure also submerged in the tank.

The source (TX) is a piston shaped projector (Panametrics A391S), with a radiation pattern concentrated around $\pm 10^{\circ}$, while the receiver $(\mathrm{RX})$ is an omni directional hydrophone (Reson TC4038). The source and the receiver were fixed to a rigid arm, driven by a rotary stage (Vemex B4836TS) that varies the angle of incidence $\theta_{i}$. The angle- and frequencydependent volume scattering was measured by transmitting linear chirps with duration of $18 \mathrm{~ms}$ in the frequency band of $250 \mathrm{kHz}$ to $450 \mathrm{kHz}$, and pulse compression was used to obtain time resolution and distinguish the returns from the scattering media, the walls of the tank and other supporting structures. In all cases, both the incident and scattered pulses were recorded at the hydrophone and compensated for spherical spreading using the lengths $L$ and $R$ indicated in Fig. 1(b).

The angle-dependent scattering from the slab was measured as follows: at each angle $\theta_{i}, N=30$ realizations were taken by laterally shifting the slab in the $\pm x$ direction (see Fig. 1(b)). This assures that each realization corresponds to a different ensemble of scatterers. The frequency dependent backscattering of the $n^{t} h$ realization for an angle of incidence $\theta_{i}$ was computed as: 


$$
\Upsilon_{n}\left(f, \theta_{i}\right)=C \frac{\left|\mathscr{F}\left(w_{n}^{s}\left(t, \theta_{i}\right)\right)\right|^{2}}{\left|\mathscr{F}\left(w_{n}^{i}\left(t, \theta_{i}\right)\right)\right|^{2}} ;
$$

where $f$ is the frequency in $\mathrm{Hz}, \mathrm{C}$ is a compensation factor for spherical spreading, $w_{n}^{s}\left(t, \theta_{i}\right)$ and $w_{n}^{i}\left(t, \theta_{i}\right)$ are the timegated scattered and direct arrivals, $\mathscr{F}$ indicates the Fourier transform and |.| is the absolute value. The obtained average backscattering $\Upsilon_{a v}\left(f, \theta_{i}\right)=(1 / N) \sum_{n=1}^{N} \Upsilon_{n}\left(f, \theta_{i}\right)$ and its standard deviation are presented in the next section and compared to simulations using the RT model.

For experiment 1 the scattering media consisted of aluminum spheres of radius $a=4.8 \mathrm{~mm}$ suspended in water, while experiment 2 utilized a resin slab of dimensions $30 \times 30 \times 5 \mathrm{~cm}$, facilitated by the Laboratory of Mechanics and Acoustics (CNRS/LMA, France). The slab contains a uniform distribution of glass beads of $1 \mathrm{~mm}$ diameter, with a fractional volume of $10 \%$. This model has been utilized in the past for similar measurements [10] at a frequency of $500 \mathrm{kHz}$, and its mechanical and acoustic properties have been well characterized. Table I summarizes the parameters of the scatterers and the background for both experiments. These values, together with the corresponding fractional volume constitute the inputs provided to the RT model.

TABLE I

ACOUSTIC PROPERTIES OF THE SCATTERING MEDIA USED IN THIS WORK. PARAMETERS FROM [11] (EXPERIMENT 1) AND [10] (EXPERIMENT 2).

\begin{tabular}{|c|c|c|c|c|}
\hline & \multicolumn{2}{|c|}{ Experiment 1} & \multicolumn{2}{c|}{ Experiment 2 } \\
\hline Variable & Background & Scatterers & Background & Scatterers \\
\hline$\rho_{1}\left(\mathrm{~kg} / \mathrm{m}^{3}\right)$ & 1000 & 2700 & 1251 & 2539 \\
\hline$c_{L 1}(\mathrm{~m} / \mathrm{s})$ & 1468 & 6290 & 1020 & 5231 \\
\hline$c_{T 1}(\mathrm{~m} / \mathrm{s})$ & 1 & 3260 & 1 & 3124 \\
\hline$\alpha_{L}(\mathrm{~Np} / \mathrm{m})$ & 80 & N/A & 0 & N/A \\
\hline
\end{tabular}

\section{RESULTS AND COMPARISON TO THE RT MODEL}

In this section, experimental results for both experiments are presented and compared to simulations using the RT model, computed from (3). As a convention, solid lines represent experimental results, while simulations are shown as dashed lines in the same color.

\section{A. Experiment 1: Aluminum spheres in water}

For this experiment only normal incidence measurements $\left(\theta_{i}=0\right)$ were considered. The spheres were suspended with nylon filament and positioned randomly to fit within a volume of $16 \times 16 \times 1 \mathrm{~cm}$, and measurements were taken for fractional volumes of $0.9 \%$ and $2.7 \%$. Scattering from the supporting filaments was negligible, as determined by preliminary measurements. From Fig. 1(b), the relative location of the source, receiver and slab are $L=18 \mathrm{~cm}$ and $R=36 \mathrm{~cm}$, which results in an illuminated patch of area $d A=0.028 \mathrm{~m}^{2}$ given the radiation pattern of the source. This value is required as an input to the RT model, as explained in section II.

Figure 3 shows $\Upsilon_{a v}\left(f, \theta_{i}=0\right)$ in the frequency band $280 \mathrm{kHz}$ to $340 \mathrm{kHz}$ with fractional volume of $0.9 \%$ and $2.7 \%$ as a parameter. The backscattering levels computed with the RT model as in (5) closely match the experimental results, and since $z_{d} \eta \kappa<<1$, the RT model converges to (7). To verify the accuracy of the measurement system, scattering measured from a single sphere is shown and compared to the theoretical frequency dependency, obtained by computing the scattering function as in [12].

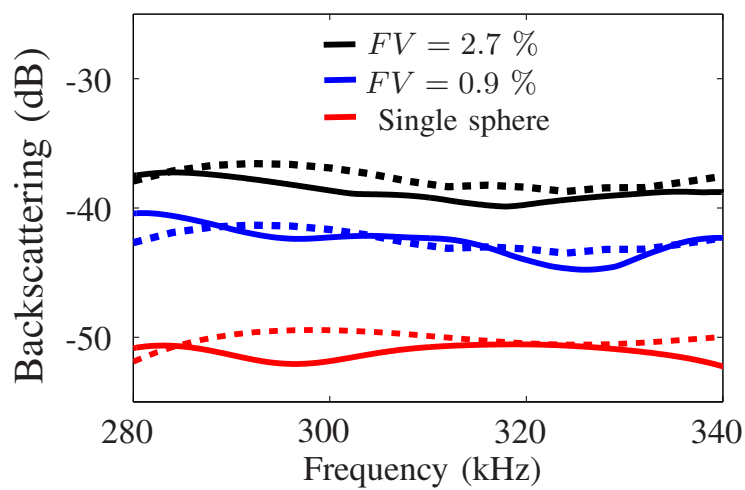

Fig. 3. Comparison of the measured backscattering $\Upsilon_{a v}\left(f, \theta_{i}=0\right)$ (solid lines), with computations from the RT model for an ensemble of aluminum spheres, for two values of fractional volume (FV). As a reference, experimental and theoretical scattering from a single sphere are also shown.

\section{B. Experiment 2: Glass beads in resin}

As mentioned in the introduction, this experiment was a better opportunity to portray the flexibility of the RT model due to more challenging conditions. In this experiment, $L=$ $25 \mathrm{~cm}, R=15 \mathrm{~cm}$, and the angle of incidence $\theta_{i}$ was varied from $0^{\circ}$ to $75^{\circ}$ in steps of $5^{\circ}$.

Fig. 4(a) shows pulse-compressed realizations corresponding to two scattering angles. At normal incidence $\left(\theta=0^{\circ}\right)$, the scattered energy includes both the specular reflection from the water-slab interface and the contribution from the scatterers, while at $\theta=10^{\circ}$ the return is due only to volume scattering. Figure 4(b) shows the corresponding $\Upsilon_{a v}(f, \theta)$, with thinner solid lines that indicate \pm 1 standard deviation. The large standard deviation and frequency dependent variability of the mean required further analysis to determine if artifacts are being introduced in the data by taking the spectral ratio in (8). To this end, scattering from an aluminum plate temporary placed in front of the slab was measured and processed in a similar fashion. The frequency-dependent scattering from this (fairly) perfect reflector is also shown in Fig. 4(b), and it exhibits a flat response around $0 \mathrm{~dB}$ for the whole frequency band. This ruled out the existence artifacts from (8), and confirmed the correct value of the constant $C$ used to compensate for spherical spreading.

The large standard deviation of roughly $\pm 5 \mathrm{~dB}$ shown in Fig. 4(b) was present at all scattering angles, with even larger values for $\theta_{i}>40^{\circ}$. This amount of variability was also observed in experimental and simulated data by Canepa et al [10] at $500 \mathrm{kHz}$, and it will be discussed in section V.

Fig. 5 shows volume scattering measured at $300 \mathrm{kHz}$ and $400 \mathrm{kHz}$ (solid lines). The mean value of the backscattered 

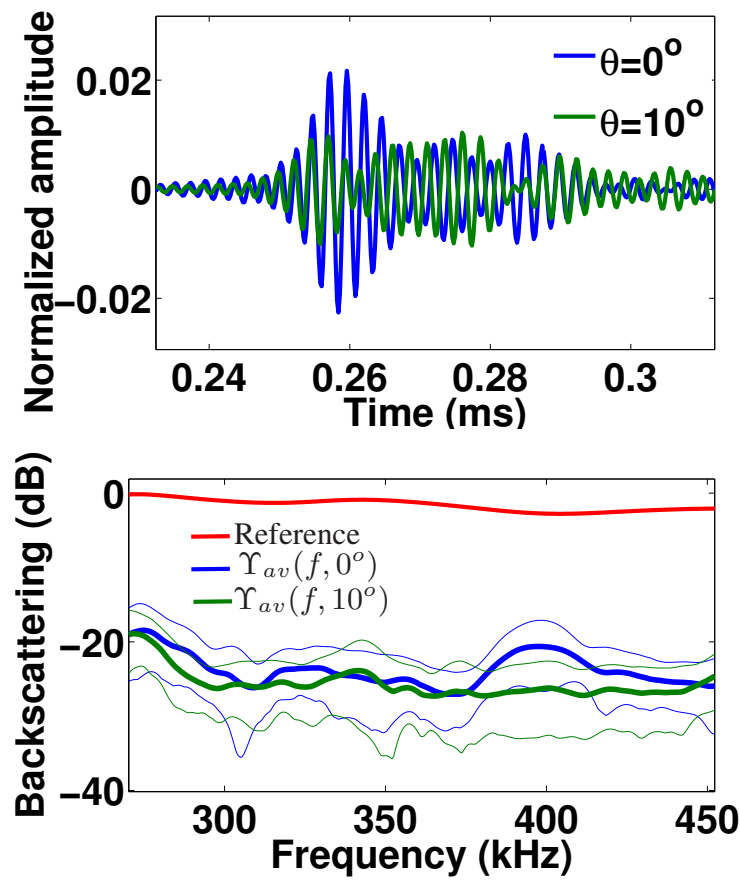

Fig. 4. (a) Example of two realizations of the backscattered signal after pulse compression, for $\theta=0^{\circ}$ and $\theta=10^{\circ}$. The waveforms are normalized to the peak value of the direct blast. (b) $\Upsilon_{a v}\left(f, \theta_{i}\right)$ for the two incident angles in (a), with thin solid lines indicating \pm 1 standard deviation around the mean of 30 realizations.

data, $\Upsilon_{a v}(f, \theta)$, exhibits angle-dependent variations and behavior similar to the measurements at $500 \mathrm{kHz}$ [10], and due to the large variability noted before, it is not possible to make a clear distinction between the scattering at different frequencies.

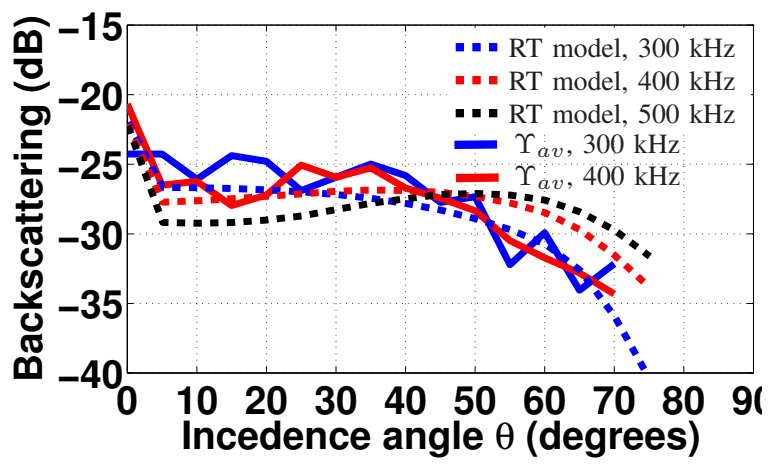

Fig. 5. Comparison of the measured backscattering, $\Upsilon_{a v}(f, \theta)$ (solid lines), with computations from the RT model at $300 \mathrm{kHz}$ and $400 \mathrm{kHz}$ (dashed lines). Computation of backscattering at $500 \mathrm{kHz}$ is also shown (black, dashed line) for comparison to experimental and simulated results by Canepa et al [10].

Simulations using the steady state RT model are shown with dashed lines for 300, 400 and $500 \mathrm{kHz}$. At $500 \mathrm{kHz}$, previous work has concluded that $\alpha_{L}=80 \mathrm{~Np} / \mathrm{m}$ is a reasonable value for the attenuation of the resin matrix. Using this value in the RT model results in scattering levels comparable to those previously reported [10]. The background attenuation at lower frequencies was not measured for this material, and it was used as a free parameter in the RT simulations in Fig. 5. At $300 \mathrm{kHz}$ and $400 \mathrm{kHz}$, attenuation coefficients of $50 \mathrm{~Np} / \mathrm{m}$ and $65 \mathrm{~Np} / \mathrm{m}$ respectively yield a good fit of the model to the mean value of the experimental data.

It is important to note that due to the small size of the slab, measurements at shallow angles were affected by edge effects of the slab as well as by scattering from the supporting structure depicted in Fig. 2, located within the tank and used to hold the slab in position. To estimate the effect of this structure, measurements were taken without the slab at all angles, and this revealed that significant contamination of the data occurred for $\theta>40^{\circ}$. This was evident from the data, which presented increasing standard deviations (not shown in Fig. 5) at larger angles of incidence.

\section{DisCUSSION AND CONCLUSIONS}

The experiments presented in this paper show the adaptability of the radiative transfer formulation for a variety of experimental conditions, including the effect of reflective boundaries and a wide frequency range. Experiment 1 was utilized as a calibration for the measurement system due to its high SNR. This experiment allowed variation of the fractional volume step by step, starting from a single scatterer. The obtained results can be verified by approximate solutions as in (7), which has already been used for analysis of field experimental data from a layer of trapped gas bubbles [9].

Results from experiment 2 evaluate the RT model in the Mie regime at a higher fractional volume and including boundary conditions. The scattering media in this experiment has two advantageous features for validation experiments: first, the acoustic impedance is matched to fresh water, increasing energy penetration and favoring volume scattering. Second the background compressional sound speed is slower than in water, allowing to test the model in the presence of refraction at the water-slab interface.

The large standard deviation observed in the experimental measurements for $\theta_{i}>40^{\circ}$ has been justified in section IV(b) as the result of data contamination. Nevertheless, the standard deviation of around $5 \mathrm{~dB}$ for $\theta_{i}<40^{\circ}$ deserves further explanation. In previous work by Canepa et al [10], scattering from the same material was studied using a time domain model that generates realizations of the ensemble of scatterers at each incident angle, and the simulated data exhibits similar behavior. This suggests that the variability observed in the experimental data is caused by frequency dependent characteristics of the ensemble of glass beads rather than by sources of experimental uncertainty. This also indicates that $\Upsilon_{a v}\left(f, \theta_{i}\right)$ can be smoothed by incorporating more realizations.

In the RT simulations for the resin slab, the background attenuation was used as a free parameter, since direct measurements are not possible due to the thickness of the slab. An alternative for estimation of the background attenuation is by running time domain simulations of backscattering [10] with attenuation as a parameter, with the goal of matching the shape of the scattered waveform to experimental measurements. The 
possibility of using this technique, as well as the simulation of short excitation pulses motivates the ongoing study of the time domain solution of (1), which can be obtained by expressing the time derivative as $\frac{\partial I(\mu, \phi, z)}{\partial t}=j \Omega I(\mu, \phi, z)$. Here, $\Omega$ is the rate of change of the specific intensity, expected to be much lower than the frequency of the excitation source. This transient RT formulation has been the subject of recent developments in modeling the propagation of fast laser excitation pulses through random media [13], and also in ultrasound characterization of materials [14]. It is still an open question whether the resulting time-dependent diffuse intensity will resemble the envelope of the instantaneous power in experimental data, which would make the time domain solution suitable as an inversion technique.

\section{ACKNOWLEDGMENTS}

This research is founded by the Office of Naval Research, ONR Award \# N000140610199.

The authors would also like to thank Dr. Jean Pierre Sessarego (Laboratory for Mechanics and Acoustics, Centre National de la Recherche Scientifique, Marseille, France) for providing the substrate utilized for some of the experimental work presented in this paper.

\section{REFERENCES}

[1] D.R.Jackson and M.D. Richardson. High Frequency Seafloor Acoustics. Springer, 2007.

[2] P. Mourad and D. Jackson. A model data comparison for low-frequency bottom backscatter. J. Acoust. Soc. Am, 94(1):344-358, 1993.

[3] A. Lyons, The potential impact of shell fragment distributions on high frequency seafloor backscatter. IEEE J. Oceanic Engineering, 30(4):843-851, 2005.

[4] A. Ishimaru, Wave Propagation and Scattering in Random Media, Volume 1, 1st ed. (Academic Press Inc., New York, 1978).

[5] A. A. Kokhanovsky, Optics of Light Scattering Media, 1st ed. (Springer, U.K., 2001)

[6] L. Margerin, M. Campillo and B. van Tiggelen. Radiative transfer and diffusion of waves in a layered medium: new insight into coda Q. Geophysical journal international, 134 (2):596-612, 1998.

[7] J.A. Turner and R.L. Weaver. Radiative Transfer of Ultrasound. $J$. Acoust. Soc. Am, 96(6):3654-3672, December 1994.

[8] J.E.Quijano and L.M.Zurk. Radiative transfer theory applied to ocean bottom modeling. J. Acoust. Soc. Am, 126(4):1712-1723, October 2009.

[9] D. Tang, G. Jin, D.R. Jackson and K.L. Williams, "Analyses of highfrequency bottom and subbottom backscattering for two distinct shallow water environments,' J. Acoust. Soc. Am. 96(5), 2930-2936 (1994).

[10] G. Canepa, J.P. Sessarego, A. Tesei, R. Guillermin and R.J. Soukup, “A time domain model of scattering from small discrete volume particles: tank validation," Proceedings of the ASA-ECUA-Euronoise Conference, France, June 2008, 4119-4124.

[11] R. Truell, C. Elbaum, and B.B. Chick, Ultrasound methods in solid state physics, 1st ed. (Academic Press Inc., New York and London, 1969).

[12] C.F. Ying and R. Truell, "Scattering of a plane longitudinal wave by a spherical obstacle in an isotropically elastic solid,' J. App. Phys. 27, 1086-1097 (1956).

[13] T. Okutucu, Y. Yener and A. A. Busnaina, "Transient radiative transfer in participating media with pulse-laser irradiation: an approximate Galerkin solution," J. Quant. Spectros. Radiat. Transfer 103, 118-130 (2007).

[14] J. A. Turner and R. L. Weaver, "Time dependence of multiply scattered diffuse ultrasound in polycrystalline media," J. Acoust. Soc. Am. 97, 2639-2644 (1995). 